\title{
Detection of Terahertz Radiation from Submicron Plasma Waves Transistors
}

\author{
Y.M. Meziani ${ }^{1}$, E. Garcia ${ }^{1}$, J. Calvo ${ }^{1}$, E. Diez ${ }^{1}$, E. Velazquez ${ }^{1}$, \\ K. Fobelets ${ }^{2}$ and W. Knap ${ }^{3}$ \\ ${ }^{1}$ Facultad de Ciencias, Universidad de Salamanca, Salamanca \\ ${ }^{2}$ Department of Electrical and Electronic Engineering, Imperial College London, London \\ ${ }^{3}$ LC2 Laboratory, Université Montpellier 2 \& CNRS, Montpellier \\ ${ }^{1}$ Spain \\ ${ }^{2}$ United Kingdom \\ ${ }^{3}$ France
}

\section{Introduction}

Terahertz $(\mathrm{THz})$ rays are located in the spectral region $\sim 0.1-10 \mathrm{THz}(\sim 3 \mathrm{~mm}-30 \mu \mathrm{m}, 3$ $\mathrm{cm}^{-1}-300 \mathrm{~cm}^{-1}$ ) between the microwave and the infrared portion of the electromagnetic spectrum. Detection and emission of terahertz radiation bears great potential in medical diagnostics, product quality control and security screening. The attractive features of $\mathrm{THz}$ radiation for applications are: (i) They are transmitted by cloths and most packaging materials such as paper or plastics (ii) Many substances have "fingerprint" spectra in the THz range (iii) Due to its low photon energy (about one million times less than X-rays), THz radiation is non-ionizing and therefore not dangerous for human beings. These properties make $\mathrm{THz}$ systems a promising tool for different types of applications wherever detection and identification of hidden threats is the task and when human beings are to be scanned. When T-rays strike an object some of the rays pass through and others bounce back. A detector can measure the time it takes for rays to bounce back from different layers of an object. From that information, computers can produce a three dimensional image of the objects internal structure. The detectors can also identify the color shift of the reflecting rays. Every chemical and material has a unique color signature (fingerprint), so $\mathrm{THz}$ systems can determine not just what an object looks like, but what it's made of. THz rays may have dozens of practical applications, from medical imaging to wireless communications (Tonouchi, 2007). New terahertz system for scanning mails is under trial in Japan (Hoshina et al., 2009). Kawase et al. has demonstrated non-destructive terahertz imaging of illicit drugs (Kawase et al., 2003). An excellent review highlighting the importance of terahertz range in different types of applications from astronomy to spectroscopy can be found in Siegel (Siegel, 2002) and references in.

Terahertz detectors that rely on quantum transitions require cryogenic temperatures, since the thermal energy $\left(k_{B} \mathrm{~T}\right)$ needs to be smaller than the quantum transition energy $(1 \mathrm{THz} \sim 4$ 
meV $\sim 50 \mathrm{~K}$ ) to avoid thermal saturation. Development of new detectors that can operate at room temperature is of big interest for terahertz technology. Dipole photoconductive antennas were developed first and are nowadays widely used in terahertz spectroscopy system for both detection and emission of terahertz pulses (Cheville, 2008; Hoffmann \& Fülöp, 2011; Smith et al., 1988). The photoconductive antenna consists of a semiconductor material (GaAs, Low temperature-grown GaAs or silicon on saphire SOS) with a short carrier lifetime and an electrode structure with a gap of $10 \mu \mathrm{m}$. The electrode are biased to a voltage of the order of 10-50 V, resulting in high electric field strength (few $\mathrm{KV} \mathrm{cm}^{-1}$ ) across the switch. When the laser pulse hits the biased gap, free carriers are generated, and subsequently accelerated by the electric field. The rapid change in polarization induced by the ultrafast accelerations of the carriers generates a sub-picosecond electromagnetic pulse that partially propagates along the electrodes and, partially, is emitted to free space. The same physical phenomena is used to detect terahertz pulses using photoconductively gated antennas. The antenna is gated on and off by the optical pulse. Only when the laser pulse generates carriers the current flows in the direction of the THz electric field. The electric field of the terahertz wave can be measured as a function of time by scanning the time delay between the narrow gate laser pulse and the $\mathrm{THz}$ pulse.

Oscillations of the plasma waves in the channel of sub-micron transistors is one of promising tools for detection of terahertz radiations at room temperature. They present many advantages: low cost, small size, room temperature operation, and tuning of the resonant frequency by the gate voltage. The interest in the applications of plasma wave devices in the THz range started at the beginning of 90's with a pioneering theoretical work of Dyakonov and Shur (Dyakonov \& Shur, 1996) who predicted that nonlinear properties of the two-dimensional (2D) plasma in the sub-micron transistor channel can be used for detection of THz radiation. Experimental investigations has been then conducted on different types of transistors demonstrating their capabilities for detection of terahertz radiation. Resonant detection from GaAs /AlGaAs FETs ${ }^{1}$ was first reported by Knap et al. (Knap, Deng, Rumyantsev, Lü, Shur, Saylor \& Brunel, 2002) at 8 K. Later, they reported on room temperature non-resonant detection (Knap, Kachorovskii, Deng, Rumyantsev, Lü, Gaska, Shur, Simin, Hu, Asif Khan, Saylor \& C. Brunel, 2002). In 2004, it was demonstrated for the first time room temperature non resonant detection from silicon field-effect transistors (Knap et al., 2004) where the responsivity was estimated at around $200 \mathrm{~V} / \mathrm{W}$ and the Noise Equivalent Power (NEP) at around $1 \mathrm{pW} / \sqrt{\mathrm{Hz}}$ (Meziani et al., 2006; Tauk et al., 2006). THz imaging based on CMOS technology has been reported by different groups (Öjefors et al., 2009; Schuster et al., 2011). Recently, a responsivity of $80 \mathrm{KV} / \mathrm{W}$ and a NEP of $300 \mathrm{pW} / \sqrt{\mathrm{Hz}}$ were reported using an array of Si-MOSFET processed by $0.25 \mu \mathrm{m}$ CMOS technology as well as imaging at 0.65 $\mathrm{THz}$ (Lisauskas et al., 2009).

Here, we report on the detection of terahertz radiation by strained $\mathrm{Si} / \mathrm{Si}_{0.6} \mathrm{Ge}_{0.4} \mathrm{n}$-MODFETs transistors. In the second section, we introduce the plasma wave oscillation under the theory of Dyakonov and Shur and we discuss both resonant and nonresonant detection cases. The third section describes the strained silicon devices and in the fourth one we present and discuss the observed resonant and non resonant detection from our devices and we explain these detections as due to the oscillations of the plasma waves in the channel. The last section

\footnotetext{
${ }^{1}$ FET: Field Effect Transistor
} 
shows the capabilities of those devices in real applications where terahertz imaging using the strained Silicon devices are presented. All those results demonstrate the ability of plasma wave devices to be used in applications whenever detection of $\mathrm{THz}(0.1-10 \mathrm{THz})$ radiation is needed.

\section{Principle of detection of terahertz radiation}

When the electron plasma in the channel of a field-effect transistor is excited by an external electromagnetic radiation the induced ac electric field can be converted into measurable dc voltage (signal) via a nonlinear conversion mechanism. This signal has a resonant dependence on the incoming radiation with maxima at the plasma oscillation frequency, $\omega_{0}$ and its odd harmonics Dyakonov \& Shur $(1995 ; 2001) \omega_{N}=(1+2 N) \omega_{0}$, where:

$$
\omega_{0}=\frac{\pi s}{2 L}
$$

$L$ is the gate length, and $s$ the plasma waves velocity which depends on the carrier density in the channel $n_{s}$, and on the gate-to-channel capacitance per unit area $C$ :

$$
s=\sqrt{\frac{e^{2} n_{s}}{m C}}
$$

where $e$ is the absolute value of the electron charge and $m$ is the electron effective mass. The surface carrier concentration $\left(n_{s}\right)$ in the channel is related to the gate-to-channel voltage swing or overdrive voltage $\left(U_{0}\right)$ by (Dyakonov \& Shur, 2001):

$$
n_{s}=\frac{C U_{0}}{e}
$$

Here $U_{0}=U_{g}-U_{t h}, U_{g}$ is the gate-to-channel voltage, and $U_{t h}$ is the threshold voltage at which the channel is completely depleted. Note that Eq. 3 is valid as long as the scale of the spacial variation of $U(x)$ is larger than the gate-to-channel separation (the gradual channel approximation). From Eqs. 1, 2, and 3, the fundamental plasma frequency can then be roughly expressed by the relation:

$$
f_{0}=\frac{1}{4 L} \sqrt{\frac{e U_{0}}{m}}
$$

This relation lead to two important consequences: (i) a submicron FET can operate as a terahertz detector (ii) the resonant frequency can be tuned by the gate bias.

The equations describing the 2D plasmon are the relationship between the surface carrier concentration and the swing voltage (Eq. 3), the equation of motion (Eq. 5), and the continuity equation (Eq. 6). The equation of motion (the Euler equation) is given by (Dyakonov \& Shur, 2001):

$$
\frac{\partial v}{\partial t}+\frac{e}{m} \frac{\partial U_{0}}{\partial x}+\frac{v}{\tau}=0
$$

where $\partial U_{0} / \partial x$ is the longitudinal electric field in the channel, $v(x, t)$ is the local electron velocity, the last term is the viscosity and accounts for electronic collisions with phonons and/or impurities, and $\tau$ is the relaxation time. Equation 5 has to be solved together with 
the continuity equation which can be written as:

$$
\frac{\partial U_{0}}{\partial t}+\frac{\partial\left(U_{0} v\right)}{\partial x}=0
$$

According to Dyakonov and Shur (Dyakonov \& Shur, 1996; 2001), the solution of those equations under the boundary conditions of common-source/open-drain is given by:

$$
\Delta U=\frac{U_{a}^{2}}{4 U_{0}} f(\omega)
$$

where $\Delta U$ is the source-to-drain voltage induced by the incoming radiation which is approximated by $U_{a} \cos (\omega t)($ Dyakonov \& Shur, 1996) and:

$$
f(\omega)=1+\beta-\frac{1+\beta \cos \left(2 k_{0}^{\prime} L\right)}{\sinh ^{2}\left(k_{0}^{\prime \prime} L\right)+\cos ^{2}\left(k_{0}^{\prime} L\right)}
$$

Here

$$
\begin{aligned}
\beta & =\frac{2 \omega \tau}{\sqrt{1+(\omega \tau)^{2}}} \\
k_{0}^{\prime} & =\frac{\omega}{s} \sqrt{\frac{\left(1+(\omega \tau)^{-2}\right)^{1 / 2}+1}{2}} \\
k_{0}^{\prime \prime} & =\frac{\omega}{s} \sqrt{\frac{\left(1+(\omega \tau)^{-2}\right)^{1 / 2}-1}{2}}
\end{aligned}
$$

Equations 7 and 8 describe the response of the device as a THz detector for any frequency and gate length. The function $f(\omega)$ depends on two dimensionless parameters: $\omega \tau$ and $s \tau / L$. Figure 1 shows $f(\omega)$ as a function of $\omega \tau$ for different values of $s \tau / L$. When $\omega \tau \gg 1$ and for submicron devices, such that $s \tau / L \gg 1, f(\omega)$ exhibit sharp resonances at the fundamental frequency and its odd harmonics (Fig. 1(a) and (b)). In this case the damping of the plasma waves excited by incoming radiation is small and the device exhibits a resonance detection mode. However, when $\omega \tau \ll 1$, the plasma oscillations are overdamped. For a long device, the oscillations excited at the source by the incoming radiation do not reach the drain because of the damping. The boundary conditions at the drain are irrelevant in this case, and the response does not depend on L. As it can be seen in Fig. 1(c) and (d), $f(\omega)$ changes from $f=1$ for $\omega \tau \ll 1$ to $f=3$ for $\omega \tau \gg 1$, where we also see how at very small values of $\omega \tau$ the condition of a long sample is violated and $f$ tends to zero. In both cases, a long channel acts as a broadband detector of electromagnetic radiation. Underdamped $\omega \tau \gg 1$ or overdamped $\omega \tau \ll 1$ plasma waves decay near the source end of the channel, leading to a dc voltage induced between drain and source.

\section{Devices description}

The epistructure of the MODFET was grown by molecular beam epitaxy (MBE) on a thick relaxed SiGe virtual substrate grown by low-energy plasma-enhanced chemical vapor deposition (LEPECVD) over a p-doped conventional Si wafer. The final Ge molar 


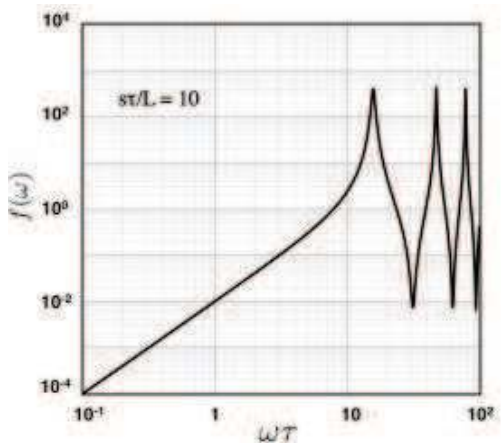

(a) $s \tau / L=10$

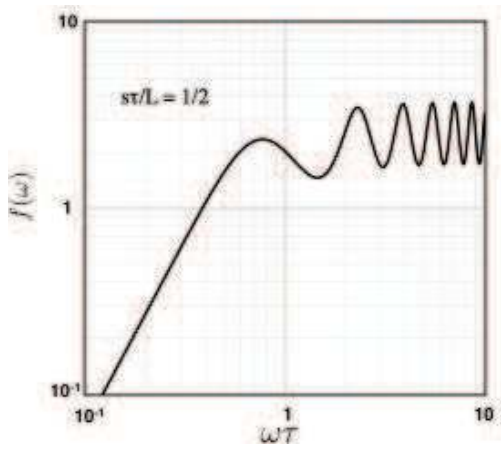

(c) $s \tau / L=1 / 2$

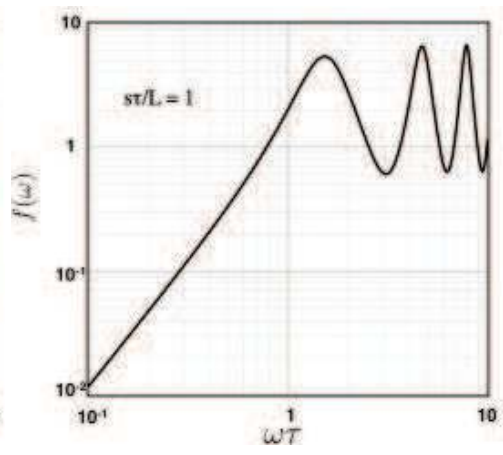

(b) $s \tau / L=1$

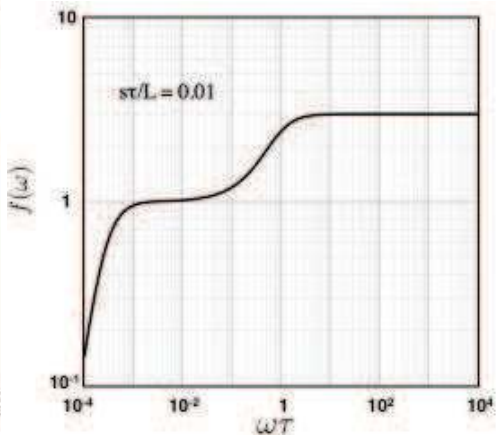

(d) $s \tau / L=0.01$

Fig. 1. Function $f(\omega)$ versus $\omega \tau$ for different values of $s \tau / L$.

concentration in the virtual substrate was $x_{G e}=0.45$. The device had a $8 \mathrm{~nm}$ tensile strained (in terms of biaxial deformation) Si channel sandwiched between two heavily doped $\mathrm{SiGe}$ electron supply layers to generate a high carrier density in the strained-Si quantum well (Rumyantsev et al., 2008). The ohmic contacts were not self-aligned. Two transistors were measured with different gate lengths $(150 \mathrm{~nm}$ and $250 \mathrm{~nm})$. The gate width and the source-to-drain length are $30 \mu \mathrm{m}$ and $1 \mu \mathrm{m}$ respectively. Figure 2 shows an image of three devices with $(50 \mathrm{~nm}, 150 \mathrm{~nm}$, and $250 \mathrm{~nm})$ gate length with common drain and different source pads.

\section{Results and discussion}

\subsection{Resonant detection}

Figure 3(a) shows the photoresponse signal as a function of the gate bias for strained Silicon transistor excited with electromagnetic radiation at 323 and $360 \mathrm{GHz}$. The gate length is 250 $\mathrm{nm}$ and the measurement was performed at $4 \mathrm{~K}$. A clear shift of the response's peak to higher gate voltage values with the frequency of the excitation was observed. The square and triangle symbols in Fig. 3(b) mark the maximum of signals at 363 and $323 \mathrm{GHz}$ respectively. According 


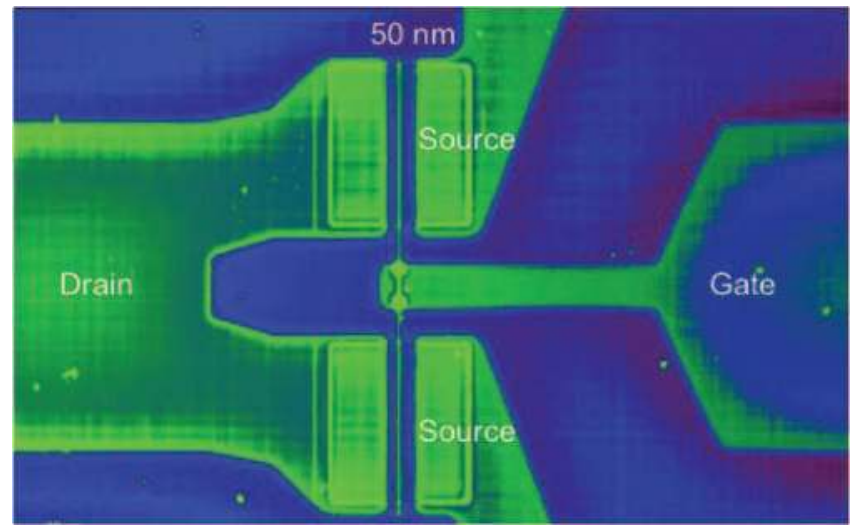

Fig. 2. Image of strained Silicon MODFET with $50 \mathrm{~nm}$ gate length. The image was captured using a 3D Laser Microscope Olympus LEXT OLS4000.

to Eq. 4, the resonant frequency as a function of the voltage swing is plotted for $\mathrm{Lg}=230 \mathrm{~nm}$ Fig. 3(b) and the observed displacement towards higher values of the gate voltage is in good agreement with Dyakonov-Shur prediction.
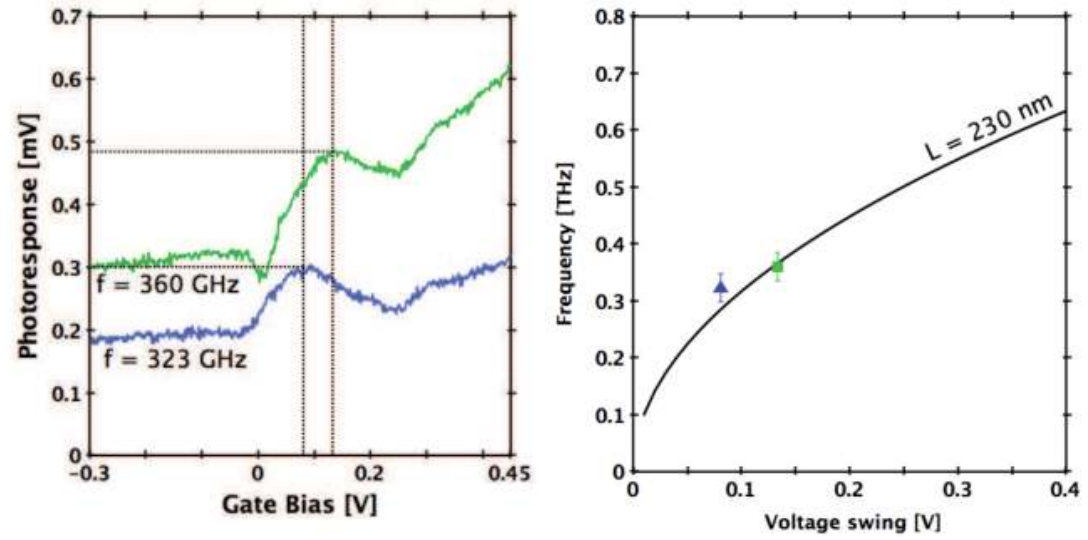

Fig. 3. (a) Photoresponse vs gate bias at two frequencies of the incoming radiation ( 323 and $360 \mathrm{GHz}$ ). (b) Resonance frequency vs swing voltage for $\mathrm{Lg}=230 \mathrm{~nm}$ gate lengths $(200,230$, and $260 \mathrm{~nm}$ ).

In the presence of a magnetic field, Eq. 7 can be written in the following form (Lifshits \& Dyakonov, 2009):

$$
\Delta U=\frac{1}{4} \frac{U_{a}^{2}}{U_{0}}\left[f(\beta)-\frac{d \gamma}{d n} \frac{n}{\gamma} g(\beta)\right]
$$

where $\beta=\frac{\omega_{c}}{\omega}$ ( $\omega_{c}$ and $\omega$ are the cyclotron resonance and the incoming radiation frequency respectively) is a normalized cyclotron resonance. Dependence of the photoresponse on the 
magnetic field and the radiation frequency is described by the functions $f(\beta)$ and $g(\beta)$ :

$$
\begin{aligned}
f(\beta) & =1+\frac{1+F}{\sqrt{\left(\alpha^{2}+F^{2}\right)}} \\
g(\beta) & =\frac{1+F}{2}\left(1+\frac{1+F}{\sqrt{\left(\alpha^{2}+F^{2}\right)}}\right) \\
F & =\frac{1+\alpha^{2}-\beta^{2}}{1+\alpha^{2}+\beta^{2}} \text { and } \alpha=(\omega \tau)^{-1}
\end{aligned}
$$

The $\gamma$ factor is an oscillating function of the magnetic field and the electron concentration that can be correlated with the Shubnikov de Haas oscillations. The second term in the right-hand side of Eq. 12, proportional to $d \gamma / d n$, is an oscillating function of the gate voltage and the magnetic field. The derivative in Eq. 12 induces a shift of $\pi / 2$ between the SdH oscillations and the photoresponse. This result has been previously observed on InGaAs FETs (Klimenko et al., 2010) and here we report the same phenomenon on Si/SiGe MODFET (Fig. 4) for the first time.

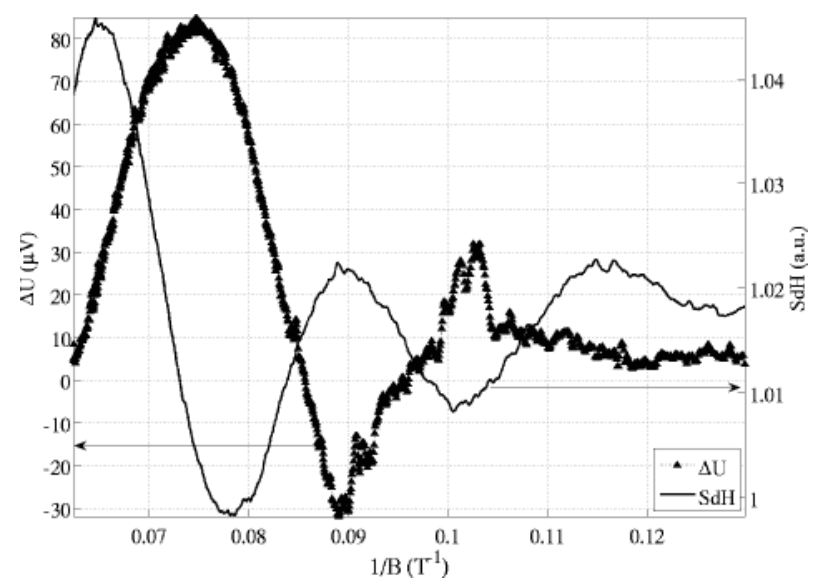

Fig. 4. Photoresponse (dashed line with triangles) and the oscillating part of the magnetoresistance (solid line) vs the inverse of the magnetic field.

\subsection{Non resonant detection}

The devices were excited at room temperature by a terahertz wave parametric oscillator (TPO) pulsed laser at $1.5 \mathrm{THz}$ (Minamide et al., 2009). The TPO system consists of three mirrors and a $\mathrm{MgO}: \mathrm{LiNbO}_{3}$ crystal under non-collinear phase-matching conditions. It can emit monochromatic THz-waves over a wide tunable frequency range from $0.4 \mathrm{THz}$ to $2.8 \mathrm{THz}$ with a narrow line-width lower than $100 \mathrm{MHz}$. The output power of the laser was $6 \mathrm{~nJ} /$ pulse for the range 1.3-1.6 THz and the repetition rate was $500 \mathrm{~Hz}$. The radiation was coupled to the dice via the metallization pads. The source terminal was grounded. The radiation intensity was modulated by a mechanical chopper at $1.29 \mathrm{KHz}$ and the induced photoresponse signal is measured by using the lock-in amplifier technique (Fig. 5). A wire grid polarizer was used to 
polarize the light in parallel with the channel. The principle of operation is as follows: when the device is excited by an external electromagnetic radiation the induced ac electric fields can be converted into a measurable dc signal via a nonlinear conversion mechanism. This will be referred hereafter as the photoresponse signal.

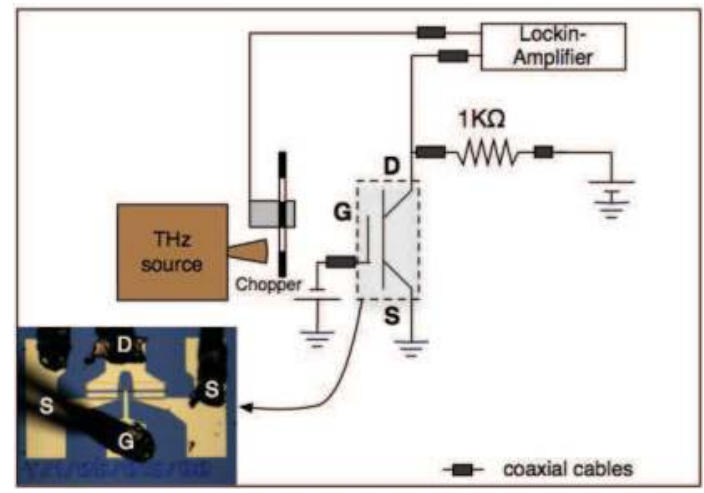

Fig. 5. Schematic description of the experimental setup of the detection of $\mathrm{THz}$ radiation.

Figure 6 shows the photoresponse signal as a function of the gate bias for two devices with different gate lengths $\left(\mathrm{L}_{G}=150\right.$ and $\left.250 \mathrm{~nm}\right)$ excited by $1.5 \mathrm{THz}$ radiation at room temperature (Meziani et al., 2011). The signal is presented for different drain currents: 20, 50 and $100 \mu \mathrm{A}$. The intensity increases with the drain current as predicted by the theory (Veksler et al., 2006) and a maximum is observed around the threshold voltage. The same behavior has been recently reported by Elkhatib et al. (2011) demonstrating that the response signal linearly increases with the drain current (or drain-to-source voltage) and the responsivity might reach high values within the saturation regime. The observed signal intensity is low because no parabolic mirror was used to focus the beam and also, as reported in Rumyantsev et al. (2008), the response intensity at terahertz frequencies (over $1 \mathrm{THz}$ ) is considerably smaller than at sub-terahertz ones (below $1 \mathrm{THz}$ ). The responsivity was estimated to be $20 \mathrm{~V} / \mathrm{J} /$ Pulse at $\mathrm{I}_{D S}$ $=100 \mu \mathrm{A}$. A non-resonant response has been reported for Si-FET Knap et al. (2004) and it was related to a low value of the quality factor i.e. low carrier mobility in the device. The quality factor was found to be around 1.2 for $\mu=1355 \mathrm{~cm}^{2} / \mathrm{V}$.s and for $f=1.5 \mathrm{THz}$. Rumyantsev et al. (2008) obtained on similar devices a maximum value of the photoresponse signal when the beam focus was away from the transistor. This is a proof of low coupling of the THz radiation to the device. To increase the efficiency toward high non-resonant signal and possible resonant detection, new designs are under consideration: array of transistors, devices with larger pads, and grating devices Popov et al. (2011a).

\section{Terahertz imaging}

First, a strained Silicon MODFET transistor with a gate length of $50 \mathrm{~nm}$ was subjected at room temperature to electromagnetic radiation at $292 \mathrm{GHz}$ from a Gunn diode (Fig. 5). Figure 7 shows the photoresponse signal as a function of the gate bias. A maximum signal is observed around the threshold voltage $(\sim-0.84 \mathrm{~V})$. This behavior has been reported earlier (Knap et al., 2004; Rumyantsev et al., 2008) and explained as non-resonant detection. However, the 

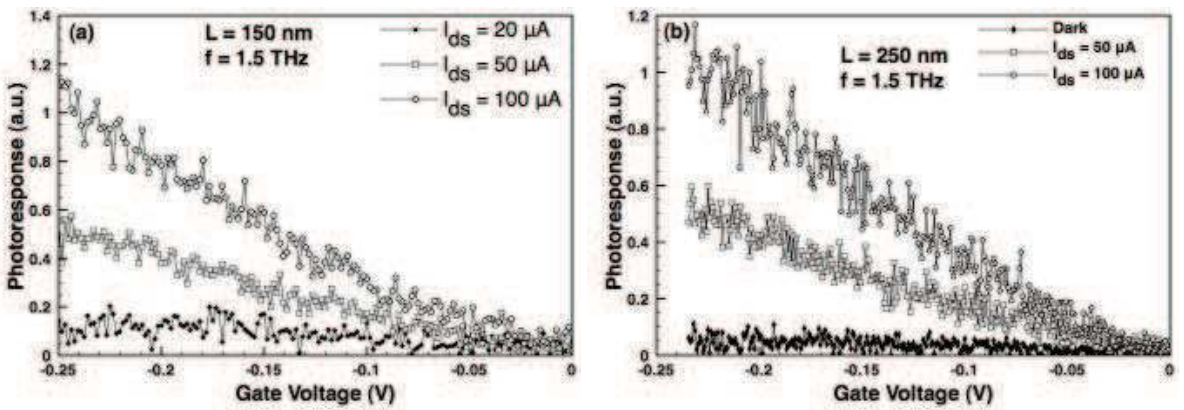

Fig. 6. Photoresponse vs gate bias at $1.5 \mathrm{THz}$ and for two devices lengths: (a) $150 \mathrm{~nm}$ and (b) $250 \mathrm{~nm}$.

signal to noise ratio shown here is high enough to allow the use of this devices for different applications like terahertz imaging. The noise equivalent power of this device is estimated around $1 \mathrm{pW} / \sqrt{\mathrm{Hz}}$ in the same order of CMOS devices. Subsequently, the device was used

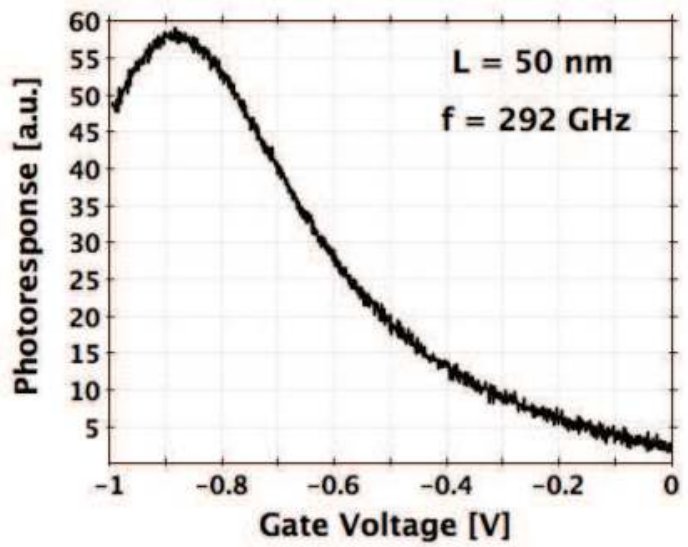

Fig. 7. Measured photoresponse of the device as function of the gate voltage. The curve shows a maximum near to the threshold voltage of the MODFET.

as a detector in a terahertz imaging system. Figure 8 describes schematically the terahertz imaging system. A Gunn diode was used as a source of terahertz radiation with $f=292 \mathrm{GHz}$. The radiation is collimated and focused by off-axis parabolic mirrors and a visible red LED in combination with an indium tin oxide (ITO) mirror are used for the alignment of the $\mathrm{THz}$ beam. The incident $\mathrm{THz}$ light is mechanically chopped at $333 \mathrm{~Hz}$ and the photo-induced drain-to-source voltage $\Delta \mathrm{U}$ is measured using a lock-in technique. All measurements were done at room temperature. More information about the $\mathrm{THz}$ imaging system can be found in Schuster et al. (2011). The gate bias $\left(\mathrm{V}_{g}\right)$ was fixed at a value close to the threshold voltage to obtain optimum signal for imaging and high values of the signal to noise ratio. Figure 9 shows both visible (left) and terahertz image (right) of a box with a hidden mirror inside. The resolution of the image is 17500 pixels within $x$ axis and of 120000 pixels within $y$ axis. 


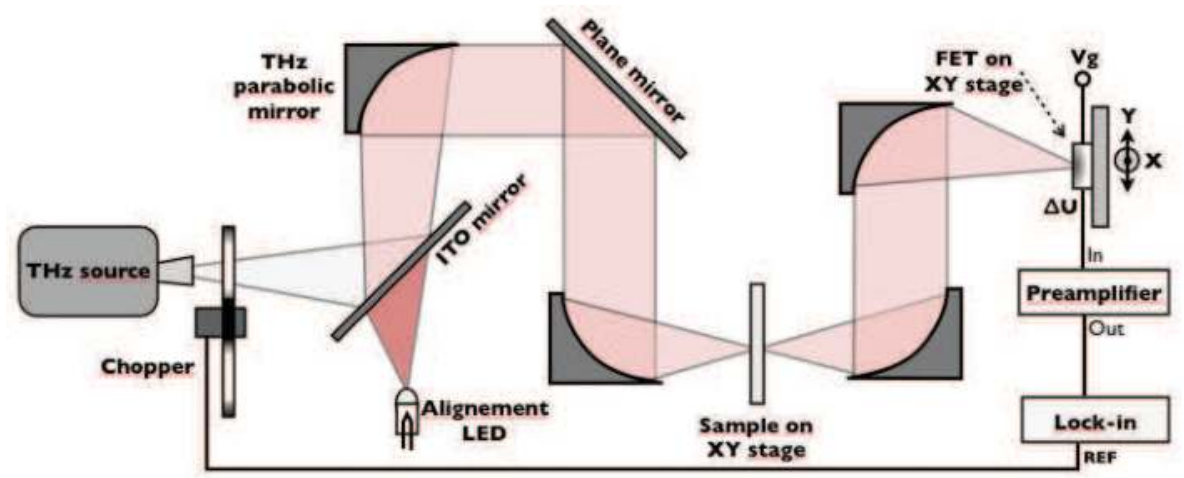

Fig. 8. Schematic description of the experimental setup of the imaging system.

Terahertz image of a VISA card is also shown in Fig. 10. The resolution of the terahertz image is $23000 \times 110000$ pixels in $\mathrm{XxY}$ axis. Those images demonstrate the capability of such devices in compact terahertz imaging systems and that they could play an important role in other $\mathrm{THz}$ applications. Those systems are inexpensive, compact, operate at room temperature and can be monolithically integrated along with Si-circuitry.
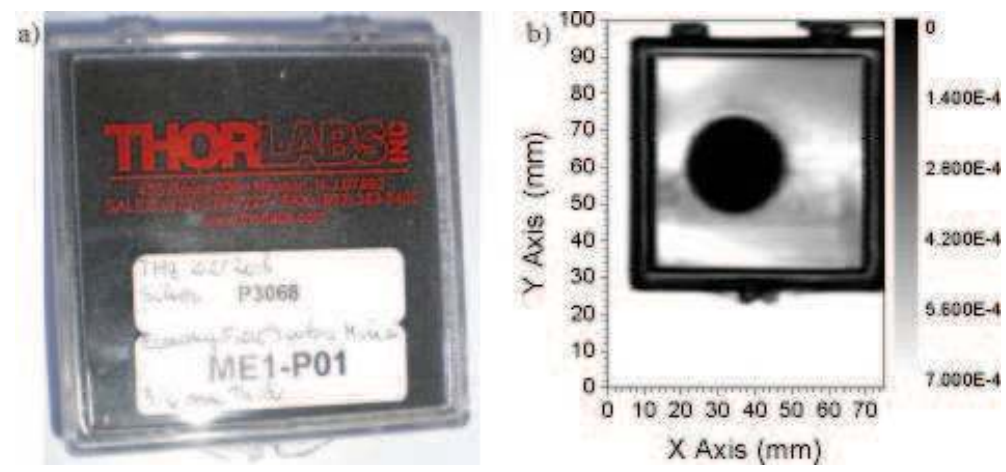

Fig. 9. (a) Visible and (b) terahertz image of a plastic box with a hidden mirror inside.

\section{Future directions}

Increasing the responsivity and the noise equivalent power are the main goals to improve the performance of the next generation of plasma wave THz detectors. This may be achieved by using new materials (like Graphene) and also by using new designs for a better coupling of the terahertz radiation into the transistor. Recently, different groups have reported record values of the NEP from different well designed devices. High responsivity $(200 \mathrm{~V} / \mathrm{W})$ and low noise equivalent power $(1 \mathrm{pW} / \sqrt{\mathrm{Hz}})$ were observed from a Si-MOSFETs with different gate lengths (120 and $300 \mathrm{~nm}$ ), Tauk et al. (2006). Terahertz imaging based on CMOS technology has been recently reported by different groups: Lisauskas et al. (2009); Schuster et al. (2011; 2010). A 3x5 Si MOSFET focal-plane array processed using conventional $0.25 \mu \mathrm{m}$ CMOS technology was used by Lisauskas et al. (2009) for imaging at $0.65 \mathrm{THz}$. Responsivity of $80 \mathrm{KV} / \mathrm{W}$ and a NEP 

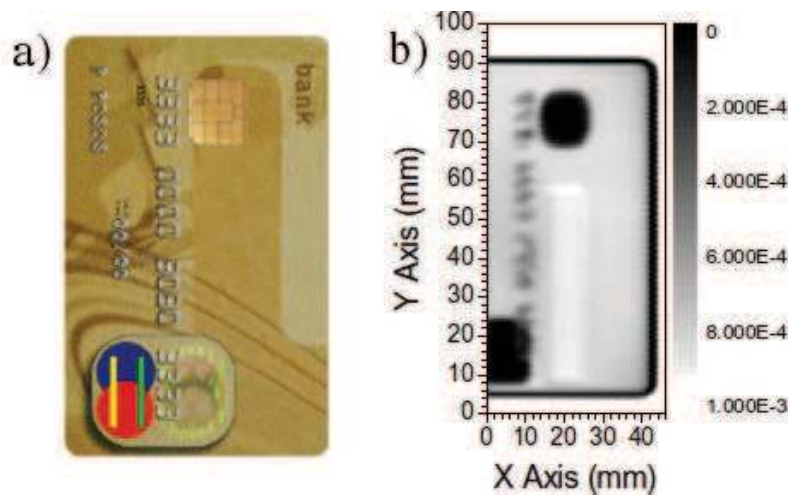

Fig. 10. (a) Visible and (b) terahertz image of a VISA card.

of $300 \mathrm{pW} / \sqrt{\mathrm{Hz}}$ was obtained. Recently, terahertz imaging was performed using a nMOS field effect transistor with an integrated bow-tie coupling antenna with a responsivity above $5 \mathrm{kV} / \mathrm{W}$ and a noise equivalent power below $10 \mathrm{pW} / \sqrt{\mathrm{Hz}}$, Schuster et al. (2011). Asymmetric doubly interdigitated grating gates structures with theoretical values of the responsivity in excess of 5 KV/W are under consideration (Otsuji et al., 3 December 2010; Popov et al., 2011b). Recently, a responsitivity up to $2.2 \mathrm{KV} / \mathrm{W}$ at $1 \mathrm{THz}$ has been reported by using asymetric grating gate structures Otsuji (November 2011).

\section{Conclusion}

We demonstrated the capability of submicron Strained $\mathrm{Si} / \mathrm{Si}_{0.6} \mathrm{Ge}_{0.4} \mathrm{n}$-MODFETs transistors for the detection of terahertz radiation. Resonant detection has been observed at low temperature and related to the plasma oscillations in the channel. A broadband (non resonant) detection has also been reported using the devices under continuous and pulsed excitation. We have shown the capabilities of those transistors in real terahertz imaging at room temperature. We believe that strained-Si transistors could play an important role in different terahertz applications in the near future.

\section{Acknowledgment}

Authors from Salamanca University acknowledge the financial help from the Ministry of Science and Innovation (MICINN) through the projects PPT-120000-2009-4, PCT-420000-2010-008 and TEC2008-02281 and Junta de Castilla y León (grants Numbers SA061A09 and SA049A10). Y.M.M. thanks the Ramon y Cajal Program for support. The authors from Montpellier University acknowledge the CNRS GDR-I project "Semiconductors Sources and Detectors of THz radiation" and the Region of Languedoc-Roussillon through the "Terahertz Platform" project.

\section{References}

Cheville, R. A. (2008). Terahertz time-domain spectroscopy with photoconductive antennas, in S. L. Dexheimer (ed.), Terahertz Spectroscopy, Taylor and Francis Group, UK, pp. 1-39. 
Dyakonov, M. \& Shur, M. (1996). Detection, mixing, and frequency multiplication of terahertz radiation by two-dimensional electronic fluid, IEEE Transactions on Electron Devices 43(3): $380-387$.

Dyakonov, M. \& Shur, M. S. (1995). Proceedings of the 22nd International Symposium on GaAs and Related Compounds, Institute Conference Series No 145, Cheju, Korea, Chap. 5, pp. 785-790 (1996).

Dyakonov, M. \& Shur, M. S. (2001). Plasma wave electronics for terahertz applications, in R. Miles, P. Harrison \& D. Lippens (eds), Terahertz Sources and Systems, Kluwer Academic Publishers, Netherlands, pp. 187-207.

Elkhatib, T. A., Kachorovskii, V. Y., Stillman, W. J., Rumyantsev, S., Zhang, X.-C. \& Shur, M. S. (2011). Terahertz response of field-effect transistors in saturation regime, Appl. Phys. Lett. 98(24): 243505.

URL: $h$ ttp://dx.doi.org/doi/10.1063/1.3584137

Hoffmann, M. \& Fülöp, J. (2011). Intense ultrashort terahertz pulses: generation and applications, Journal of Physics D: Applied Physics 44: 083001.

Hoshina, H., Sasaki, Y., Hayashi, A., Otani, C. \& Kawase, K. (2009). Noninvasive mail inspection using terahertz radiation, SPIE Newsroom .

Kawase, K., Ogawa, Y., Watanabe, Y. \& Inoue, H. (2003). Non-destructive terahertz imaging of illicit drugs using spectral fingerprints, Opt. Express 11(20): 2549-2554.

URL: http://www.opticsexpress.org/abstract.cfm?URI=oe-11-20-2549

Klimenko, O. A., Mityagin, Y. A., Videlier, H., Teppe, F., Dyakonova, N. V., Consejo, C., Bollaert, S., Murzin, V. N. \& Knap, W. (2010). Terahertz response of InGaAs field effect transistors in quantizing magnetic fields, Appl. Phys. Lett. 97(2): 022111.

URL: $h$ ttp://dx.doi.org/doi/10.1063/1.3462072

Knap, W., Deng, Y., Rumyantsev, S., Lü, J.-Q., Shur, M. S., Saylor, C. A. \& Brunel, L. C. (2002). Resonant detection of subterahertz radiation by plasma waves in a submicron field-effect transistor, Appl. Phys. Lett. 80(18): 3433-3435.

URL: $h t t p: / / d x$.doi.org/doi/10.1063/1.1473685

Knap, W., Kachorovskii, V., Deng, Y., Rumyantsev, S., Lü, J.-Q., Gaska, R., Shur, M. S., Simin, G., Hu, X., Asif Khan, M., Saylor, C. A. \& C. Brunel, L. (2002). Nonresonant detection of terahertz radiation in field effect transistors, J. Appl. Phys. 91(11): 9346-9353.

URL: $h$ ttp://dx.doi.org/doi/10.1063/1.1468257

Knap, W., Teppe, F., Meziani, Y., Dyakonova, N., Lusakowski, J., Boeuf, F., Skotnicki, T., Maude, D., Rumyantsev, S. \& Shur, M. S. (2004). Plasma wave detection of sub-terahertz and terahertz radiation by silicon field-effect transistors, Appl. Phys. Lett. 85(4): 675-677.

URL: $h$ ttp://dx.doi.org/doi/10.1063/1.1775034

Lifshits, M. B. \& Dyakonov, M. I. (2009). Photovoltaic effect in a gated two-dimensional electron gas in magnetic field, Phys. Rev. B 80: 121304.

URL: http://link.aps.org/doi/10.1103/PhysRevB.80.121304

Lisauskas, A., Pfeiffer, U., Ějefors, E., BolŞvar, P. H., Glaab, D. \& Roskos, H. G. (2009). Rational design of high-responsivity detectors of terahertz radiation based on distributed self-mixing in silicon field-effect transistors, J. Appl. Phys. 105(11): 114511.

URL: $h t t p: / / d x$.doi.org/doi/10.1063/1.3140611

Meziani, Y. M., Garcia, E., Velazquez, E., Diez, E., Moutaouakil, A. E., Otsuji, T. \& Fobelets, K. (2011). Strained silicon modulation field-effect transistor as a new sensor of terahertz 
radiation, Semiconductor Science and Technology 26(10): 105006.

URL: $h$ ttp://stacks.iop.org/0268-1242/26/i=10/a=105006

Meziani, Y. M., Lusakowski, J., Dyakonova, N., Knap, W., Seliuta, D., Sirmulis, E., Devenson, J., Valusis, G., Boeuf, F. \& Skotnicki, T. (2006). Non resonant response to terahertz radiation by submicron CMOS transistors, IEICE TRANSACTIONS on Electronics E89-C: 993-998.

Minamide, H., Ikari, T. \& Ito, H. (2009). Frequency-agile terahertz-wave parametric oscillator in a ring-cavity configuration, Rev. Sci. Instrum. 80(12): 123104.

URL: $h t t p: / / d x$.doi.org/doi/10.1063/1.3271039

Öjefors, E., Lisauskas, A., Glaab, D., Roskos, H. \& Pfeiffer, U. (2009). Terahertz imaging detectors in CMOS technology, Journal of Infrared, Millimeter and Terahertz Waves 30: 1269-1280. 10.1007/s10762-009-9569-4.

URL: $h t t p: / / d x$.doi.org/10.1007/s10762-009-9569-4

Otsuji, T., Popov, V., Knap, W., Meziani, Y., Dyakonova, N., D. Coquillat, F. T., Fateev, D. \& Perez, J. E. V. (3 December 2010). Terahertz apparatus, Japanese Patent PCT/JP2010/007074 .

Otsuji, T. (2011). Emission and detection of terahertz radiation using two-dimensional electrons in III-V semiconductors and Graphene, Proceedings of the joint conference for International Symposium on Terahertz Nanoscience (Teranano2011) and Wokshop of International Terahertz Research Network (GDR-I THz 2011), Osaka University Nakanoshima Center, Osaka, Japan, pp. 133-136.

Popov, V., Fateev, D., Otsuji, T., Meziani, Y., Coquillat, D. \& Knap, W. (2011a). Plasmonic detection of terahertz radiation in a double-grating-gate transistor structure with an asymmetric unit cell, Proceedings of XV International Symposium "Nanophysics and Nanoelectronics", Vol. 1, Institute Conference Series No 145, Nizhnii Novgorod, Russia, pp. 121-122.

Popov, V., Fateev, D., Otsuji, T., Meziani, Y., Coquillat, D. \& Knap, W. (2011b). Plasmonic terahertz detection by a double-grating-gate field-effect transistor structure with an asymmetric unit cell, Submitted to Appl. Phys. Lett.

URL: http://arxiv.org/abs/1111.1807

Rumyantsev, S. L., Fobelets, K., Veksler, D., Hackbarth, T. \& Shur, M. S. (2008). Strained-si modulation doped field effect transistors as detectors of terahertz and sub-terahertz radiation, Semiconductor Science and Technology 23(10): 105001.

URL: $h t t p: / / s t a c k s . i o p . o r g / 0268-1242 / 23 / i=10 / a=105001$

Schuster, F., Coquillat, D., Videlier, H., Sakowicz, M., Teppe, F., Dussopt, L., Giffard, B., Skotnicki, T. \& Knap, W. (2011). Broadband terahertz imaging with highly sensitive silicon CMOS detectors, Opt. Express 19(8): 7827-7832.

URL: http://www.opticsexpress.org/abstract.cfm?URI=oe-19-8-7827

Schuster, F., Sakowicz, M., Siligaris, A., Dussopt, L., Videlier, H., Coquillat, D., Teppe, F., Giffard, B., Dobroiu, A., Skotnicki, T. \& Knap, W. (2010). THz imaging with low-cost 130 nm CMOS transistors, Proc. SPIE 7837(1): 783704.

URL: $h$ ttp://dx.doi.org/doi/10.1117/12.864877

Siegel, P. (2002). Terahertz technology, IEEE Transactions on Microwave Theory and Techniques, 50(3): 910-928.

Smith, P., Auston, D. \& Nuss, M. (1988). Subpicosecond photoconducting dipole antennas, IEEE Journal of Quantum Electronics 24(2): 255-260. 
Tauk, R., Teppe, F., Boubanga, S., Coquillat, D., Knap, W., Meziani, Y. M., Gallon, C., Boeuf, F., Skotnicki, T., Fenouillet-Beranger, C., Maude, D. K., Rumyantsev, S. \& Shur, M. S. (2006). Plasma wave detection of terahertz radiation by silicon field effects transistors: Responsivity and noise equivalent power, Appl. Phys. Lett. 89(25): 253511. URL: $h$ ttp://dx.doi.org/doi/10.1063/1.2410215

Tonouchi, M. (2007). Cutting-edge terahertz technology, Nature Photonics 1: 97-105.

Veksler, D., Teppe, F., Dmitriev, A. P., Kachorovskii, V. Y., Knap, W. \& Shur, M. S. (2006). Detection of terahertz radiation in gated two-dimensional structures governed by dc current, Phys. Rev. B 73: 125328.

URL: http://link.aps.org/doi/10.1103/PhysRevB.73.125328 


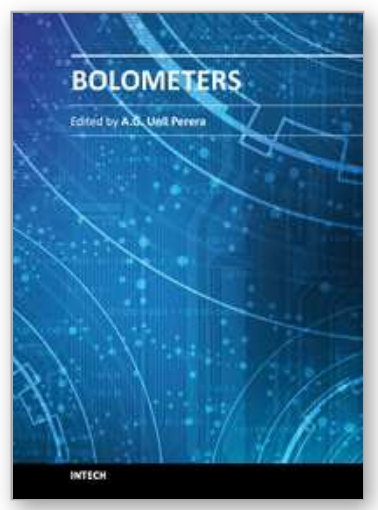

\author{
Bolometers \\ Edited by Prof. Unil Perera
}

ISBN 978-953-51-0235-9

Hard cover, 196 pages

Publisher InTech

Published online 09, March, 2012

Published in print edition March, 2012

Infrared Detectors and technologies are very important for a wide range of applications, not only for Military but also for various civilian applications. Comparatively fast bolometers can provide large quantities of low cost devices opening up a new era in infrared technologies. This book deals with various aspects of bolometer developments. It covers bolometer material aspects, different types of bolometers, performance limitations, applications and future trends. The chapters in this book will be useful for senior researchers as well as beginning graduate students.

\title{
How to reference
}

In order to correctly reference this scholarly work, feel free to copy and paste the following:

Y. M. Meziani, E. Garcia, J. Calvo, E. Diez, E. Velazquez, K. Fobelets and W. Knap (2012). Detection of Terahertz Radiation from Submicron Plasma Waves Transistors, Bolometers, Prof. Unil Perera (Ed.), ISBN: 978-953-51-0235-9, InTech, Available from: http://www.intechopen.com/books/bolometers/detection-ofterahertz-radiation-from-submicron-plasma-waves-transistors

\section{INTECH}

open science | open minds

\section{InTech Europe}

University Campus STeP Ri

Slavka Krautzeka 83/A

51000 Rijeka, Croatia

Phone: +385 (51) 770447

Fax: +385 (51) 686166

www.intechopen.com

\section{InTech China}

Unit 405, Office Block, Hotel Equatorial Shanghai

No.65, Yan An Road (West), Shanghai, 200040, China 中国上海市延安西路65号上海国际贵都大饭店办公楼 405 单元

Phone: +86-21-62489820

Fax: +86-21-62489821 
(C) 2012 The Author(s). Licensee IntechOpen. This is an open access article distributed under the terms of the Creative Commons Attribution 3.0 License, which permits unrestricted use, distribution, and reproduction in any medium, provided the original work is properly cited. 